\title{
Ruptured aneurysm of the hepatic artery: a mismatching diagnosis
}

\section{Rottura di aneurisma dell'arteria epatica: una diagnosi difficile}

\section{Vincenzo Bua, Lorenzo Marsigli*, Roberto Nardi, Anna Maria Trivella, Salvatore Isceri, Maria Luisa Piegaia, Andrea Pierfederici, Maurizio Ferretti, Luciana Boriani, Alessandra Brunori, Angelo Ziosi}

Internal Medicine-Emergency Room Department, Radiology Service, Azienda USL di Bologna

Ricevuto il 16 marzo 2009; accettato il 23 marzo 2009

disponibile online il 13 luglio 2009

\section{KEYWORDS}

Hepatic aneurysms;

Abdominal pain;

Intra-abdominal

hemorrhage;

Transcatheter

embolization.

\begin{abstract}
Summary
Background: Hepatic aneurysms are extremely rare with very few cases reported, and most have been source of misdiagnosis and clinical pitfalls in emergency medicine. Presentation with intraabdominal hemorrhage is associated with a high mortality rate.

Case report: We report the case of an adult male, referred for a severe acute pain in the left lower chest-upper quadrant abdomen pain. We present multislice contrast-enhanced CTscanning and angiographic findings, and a life-saving emergency trancatheter embolization, using fragments of absorbable gelatin sponge. Emergency doctors should consider ruptured hepatic artery aneurysms in the differential diagnosis of acute abdominal pain and promptly cooperate with endovascular specialists to treat this life-threatening condition.

(c) 2009 Elsevier Srl. All rights reserved.
\end{abstract}

\section{Introduction}

Hepatic artery aneurysms are rare clinical-pathological entities, most often discovered as an incidental finding at autopsy. Patients are often asymptomatic and late presentation may represent a difficult diagnostic challenge in emergency medical practice $[1,2]$.

* Corresponding author. Ospedale di Bazzano, v.le dei Martiri 10/B - 40053 Bazzano, BO.

E-mail: I.marsigli@gmail.com (L. Marsigli).
We report the case of an adult male, referred to our Internal Medicine-Emergency Room Observational Unit for a severe acute pain in the left lower chest-upper quadrant abdomen pain.

\section{Case report}

A fifty-one-year old man was admitted to our observational unit complaining of lower left chest-upper abdominal pain at rest. The patient, smoker with an history of ARBs treated hypertension, previous renal stones former, was to travel to 
Caribbean holidays, in "a hurry to leave, with his flight ticket in his pocket".

Initial physical examination showed a suffering looking man, owing to pain. He was not pale nor jaundiced, and his temperature was $36.5^{\circ} \mathrm{C}$. He had a blood pressure of $150 / 80 \mathrm{mmHg}$, heart rate of $76 /$ minute, and respiratory rate of $18 /$ minute. He had a substantially normal abdomen, with diffuse upper quadrants tenderness without distension. A first ECG was performed which showed sinus rhythm and a left anterior fascicular block. Laboratory findings, (CPK/MB and troponin) were normal.

After a transient relief of symptoms by analgesic therapy, the pain relapsed, and a second ECG showed inferior ST segment changes, without other enzymatic lab abnormalities. Echocardiogram showed mild mitral regurgitation but no segmental dysfunction. Chest and abdominal radiograms were normal. Cardiovascular risk factors, as well as the second ECG anomalies, rised the suspicion of atypical acute coronary syndrome, and aspirin and subcutaneous enoxaparin were initiated. Therapy was then stopped after few hours, as no further ECG/laboratory confirmations of myocardial damage have been obtained. Patient's observation carried on, with scheduled request for abdominal ultrasound (US) examination.

During observational stay, while waiting for US examination, the patient suddenly collapsed with shock: blood pressure dropped to $80 / 40 \mathrm{mmHg}$ and heart rate to $40 /$ minute, with a $\mathrm{Hb}$ from initial values of $16.1 \mathrm{~g} / \mathrm{dL}$ to $10.7 \mathrm{~g} / \mathrm{dL}$, suggesting acute haemorrhage. Emergency abdominal US was then performed, showing an anechoic right upper quadrant area without aortic or other pathological findings. Subsequent emergency contrast-enhanced CT scan showed left hepatic artery dilatation (fig. 1) and massive hemoperitoneum. The patient's hemoglobin level further decreased. The patient was suddenly transferred to the "hub" Maggiore Hospital in Bologna where an abdomen angiography was performed. Selective hepatic arteriogram (fig. 2) suggested

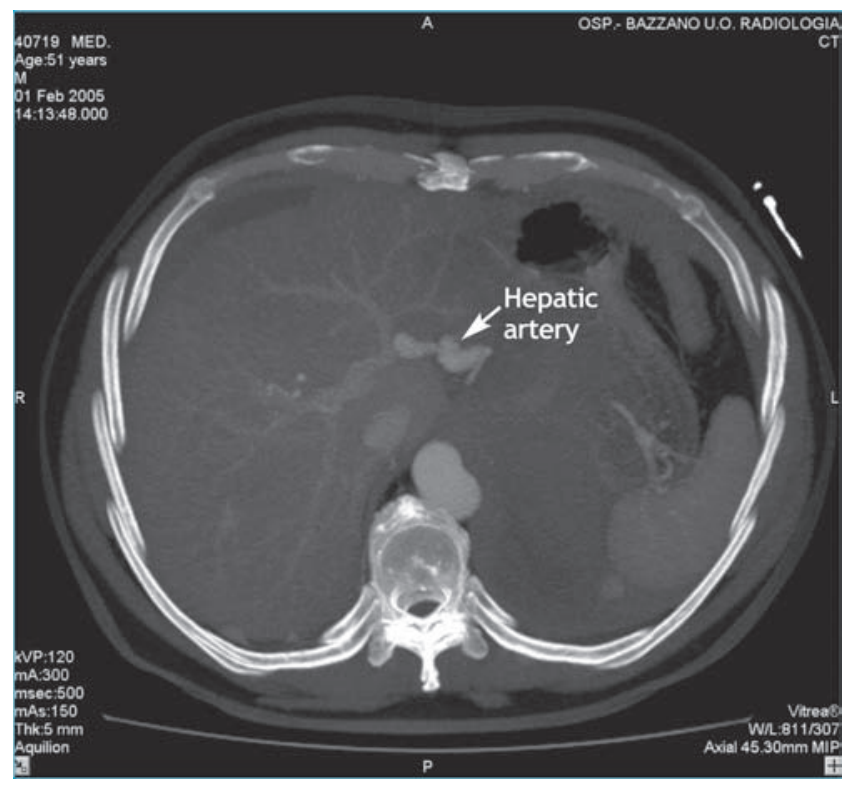

Figure 1 Contrast-enhanced CT scan shows left hepatic artery dilatation (arrow).

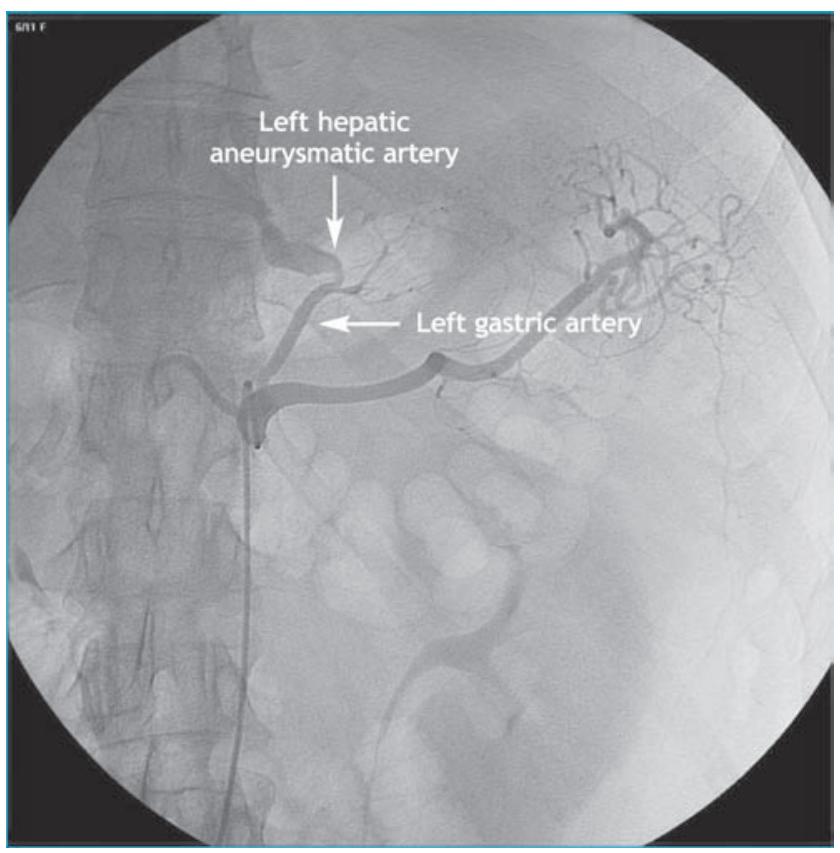

Figure 2 Selective hepatic arteriogram shows the presence of a left hepatic bleeding aneurysm. Left hepatic artery arises from left gastric artery.

a left hepatic bleeding aneurysm. As an associated finding, left hepatic artery arised from left gastric artery. A 4F Cobra glide catheter was advanced into the left gastric artery and the tip was placed in the hepatic artery aneurysm's neck. Fragments of absorbable gelatin sponge (Spongostan ${ }^{T M}$ ) were used to embolise the bleeding artery and the whole aneurysm. Haemorrhage immediately ceased, resulting in the resolution of patient's blood pressure instability. After haemostasis, late angiographic controls demonstrated no other bleeding sources. Further controls showed a good outcome. In order to obtain a permanent embolization with coils and/ or glues or with stent graft placement, angiography was repeated few days later, and no signs of bleeding were found. Moreover, it was impossible to enter into the aneurysm because of thrombosis. Our patient showed good recovery and up to-day he is still free from symptoms.

\section{Discussion}

The hepatic artery is the fourth most common site of intraabdominal aneurysms from different causes, following the infrarenal aorta, iliac and splenic arteries. True hepatic artery aneurysms must be differentiated from false (pseudo) aneurysms. Out of all patients with true hepatic artery aneurysm, $80 \%$ have been reported to develop catastrophic rupture, either into the peritoneal cavity, biliary tree, duodenum, stomach, or portal vein [3]. Eighty percent of hepatic artery aneurysms are extra-hepatic and among these, 63\% affect the common hepatic artery, $5 \%$ the left hepatic artery and $4 \%$ the right and left hepatic arteries [4]. Lesions may be single or multiple. The disease is often asymptomatic and the average age of presentation is often late in fourth and fifth decade of life, with men affected in $65 \%$ of cases [4-6]. 
Various aetiologies cause hepatic artery aneurysms. Atherosclerosis, infection (often mycotic), polyarteritis nodosa and injury account for most of these aneurysms. Pancreatitis, hereditary telangiectasias (type IV EhlersDanlos syndrome), cystic medial necrosis, vasculitis, liver abscess and tuberculosis are less common causes [7-9]. Intra-hepatic aneurysms are almost always false aneurysms related to traumatic disruption of an intraparenchymal artery. Trauma is by far the most common cause of hemobilia accounting for $50 \%$ of the cases; approximately $1 / 3^{\text {rd }}$ are iatrogenic, including diagnostic biopsy and percutaneous transhepatic cholangiography. Other less common causes are post cholecystectomy status and surgical manipulations of the biliary tree causing mucosal lacerations. Occasionally bleeding can be due to pseudoaneurysm following surgical exploration [10]. Hepatic artery pseudoaneurysm in pancreatitis is thought to occur because of the autodigestion of the arterial wall by pancreatic enzymes, especially elastase [11]. Pseudoaneurysm of the hepatic artery due to cholecystitis may be very rare [12].

Patients usually present with abdominal pain and lump, and rarely with gastrointestinal haemorrhage or obstructive jaundice $[4,5]$. On examination, abdominal bruit or a pulsatile mass may be found [9]. Aneurysms compressing a nearby bile duct may narrow or even block it, resulting in jaundice. They may also bleed into bile ducts. Anyhow, abdominal pain, hemobilia and jaundice [13], described as classical triad of presentation, are rarely seen $[4,6]$. Hemobilia due to hepatic artery aneurysms only accounted for $10 \%$ of cases [14], and was mostly due to atherosclerosis or trauma [15]. Jaundice, secondary to extraductal compression or intraductal clot, present in $50 \%$ of aneurysms ruptures [16]. Acute abdominal pain, due to the to rupture of the aneurysm into the peritoneal cavity, may also be the onset presentation. If bleeding is massive, this condition may be fatal soon.

The gold standard for diagnosis is angiography, but US, color-Doppler and contrast-enhanced CT can now reliably provide essential information for prompt decision making [9].

The treatment of choice is percutaneous embolization, surgical closure of the artery being reserved for cases unresolved by interventional procedures. Embolisation can be performed using gelfoam or steel coils [17]. Previously reported cases show that it is usually not necessary to embolize the aneurysm cavity but simply to occlude the feeding proximal vessel, therefore sparing the risk of rupture of the weak aneurysmic wall and allowing the aneurysms to clot as a result of reduced or occluded blood flow [18]. Coils are considered the optimal device to close aneurysm [17,19]. Moreover, emergency embolisations need a very high flexibility in their practice application, with modified techniques according to the patient's haemodinamic status, number of supplying vessels, flow rate of (pseudo)aneurysms, vascular anatomy and whether there is clinical evidence of re-bleeding or not [20]. Due to the extreme instability of the patient we used Spongostan ${ }^{T M}$ to stop bleeding in emergency, and we obtain a surprisingly good result. In fact, controls performed after three days, one week and six months, showed no Doppler signals into the aneurysm, and delayed angio-CT confirmed this outcome.

\section{Conclusions}

Hepatic aneurysms are extremely rare with very few cases reported, and most have been source of misdiagnosis and clinical pitfalls in emergency medicine. Their rarity is the reason why they are almost always not included in the differential diagnosis and in the diagnostic workout of the abdominal pain and/or masses. US study, as the first emergency examination after clinical evaluation, should be immediately followed by contrast-enhanced-CT and by therapeutic angiography, which is actually the gold standard for treatment. Surgical hepatic artery ligation has also been performed, but often with poor results. Moreover, vascular surgical attempts are often offset by underlying disseminated intravascular coagulopathy, which can aggravate the bleeding.

\section{Conflict of interest statement}

The authors have no conflict of interest.

\section{References}

[1] Sgroi G, Stringhi E, Bergamaschi E, Ghilardi G, Scorza R. A case of asymptomatic giant aneurysm of the common hepatic artery. J Cardiovasc Surg (Torino) 1994;35(4):337-9.

[2] Halaby H, Al-Mehaidib A, Al-Otaibi L, Al-Nassar S, Rossi L. Ruptured hepatic artery aneurysm in a child. Ann Saudi Med 2000;20(5-6):427-9.

[3] Cahow CE, Gusberg RJ, Gottlieb LJ. Gastrointestinal hemorrhage from pseudoaneurysms in pancreatic pseudocysts. Am J Surg 1983;145(4):534-41.

[4] Guida PM, Moore SW. Aneurysm of the hepatic artery. Report of five cases with a brief review of the previously reported cases. Surgery 1966;60(2):299-310.

[5] Croom 3rd RD, Frantz PT, Thomas Jr CG, Hothem AL. Aneurysms of the hepatic artery. South Med J 1976;69(8):1013-6.

[6] Gore RM. Vascular disorders of the liver and splanchnic circulation. In: Gore RM, Levine MS, Laufer I, editors. Textbook of Gastrointestinal Radiology, Vol 2. Philadelphia, PE: WB Saunders; 1994. p. 2048-50.

[7] Deol PS, Gopinath RG, Elangovan S, Anil K. Case report: Duplex imaging of a tuberculous etiology common hepatic artery aneurysm causing hematemesis. Indian J Radiol Imaging 1999;9: 13-5.

[8] Salo JA, Aarnio PT, Järvinen AA, Kivilaakso EO. Aneurysms of the hepatic arteries. Am Surg 1989;55(12):705-9.

[9] Parmar H, Shah J, Shah B, Patkar D, Varma R. Imaging findings in a giant hepatic artery aneurysm. J Postgrad Med 2000;46(2): 104-5.

[10] Garg A. Endovascular treatment of hepatic artery pseudoaneurysm complicating cholecystectomy. Case 7. http://www.kem. edu/dept/radiology/inter_7.htm.

[11] Burke JW, Erickson SJ, Kellum CD, Tegtmeyer CJ, Williamson BR, Hansen MF. Pseudoaneurysms complicating pancreatitis: detection by CT. Radiology 1986;161(2):447-50.

[12] Akatsu T, Hayashi S, Egawa T, Doi M, Nagashima A, Kitano M, et al. Hepatic artery pseudoaneurysm associated with cholecystitis that ruptured into the gallbladder. J Gastroenterol 2004; 39(9): 900-3.

[13] Zachary K, Geier S, Pellecchia C, Irwin G. Jaundice secondary to hepatic artery aneurysm: radiological appearance and clinical features. Am J Gastroenterol 1986;81(4):295-8.

[14] Harlaftis NN, Akin JT. Hemobilia from ruptured hepatic artery aneurysm. Report of a case and review of the literature. Am J Surg 1977;133(2):229-32. 
[15] Liu TT, Hou MC, Lin HC, Chang FY, Lee SD. Life-threatening hemobilia caused by hepatic artery pseudoaneurysm: A rare complication of chronic cholangitis. World J Gastroenterol 2003;9(12):2883-4.

[16] Lewis Jr DR, Kung H, Connon JJ. Biliary obstruction secondary to hepatic artery aneurysm: cholangiographic appearance and diagnostic considerations. Gastroenterology 1982;82(6):1446-51.

[17] Herskowitz MM, Flyer MA, Sclafani SJ. Percutaneous transhepatic coil embolization of a ruptured intrahepatic aneurysm in polyarteritis nodosa. Cardiovasc Intervent Radiol 1993;16(4): 254-6.
[18] Uflacker R. Transcatheter embolisation of arterial aneurysms. Br J Radiol 1986;59(700):317-24.

[19] Stambo GW, Guiney MJ, Cannella XF, Germain BF. Coil embolization of multiple hepatic artery aneurysms in a patient with undiagnosed polyarteritis nodosa. J Vasc Surg 2004;39(5): $1122-4$.

[20] Lau KY, Wong TP, Wong WW, Chan JK, Kan WK, Chan YF, et al. Transcatheter embolisation of visceral pseudoaneurysms: technical difficulties and modification of embolisation technique. Eur J Vasc Endovasc Surg 2005;30(2):133-6. 